\title{
Germinability of pioneer plant species from Mediterranean mountains occurring on screes and debris
}

\begin{abstract}
Carruggio, F., Castrogiovanni, M., Impelluso, C. \& Cristaudo, A.: Germinability of pioneer plant species from Mediterranean mountains occurring on screes and debris [In Magrini, S. \& Salmeri, C. (eds), Mediterranean plant germination reports - 2]. Fl. Medit. 30: 408-414. 2020. http://dx.doi.org/10.7320/FlMedit30.408

The present work focuses on germination requirements of four pioneer plant species from Mediterranean mountains, mainly occurring on more or less consolidated limestone screes and debris. The study species are Aethionema saxatile subsp. saxatile, Arenaria grandiflora subsp. grandiflora, Iberis violacea, and Odontarrhena nebrodensis subsp. nebrodensis. Seeds were collected at the time of natural dispersal, on the Madonie Massif, within an altitudinal range of 1300-1500 m a.s.1. Different germination conditions were tested and the best germination protocols for each species are provided.
\end{abstract}

Key words: germination protocols, high-mountain habitats, environmental constraints, Madonie Massif.

\section{Introduction}

The present study examined the germination behavior of four phytogeographically significant taxa mainly occurring on Mediterranean mountains. More specifically, we provide the first germination data for Aethionema saxatile (L.) R. Br. subsp. saxatile, Arenaria grandiflora L. subsp. grandiflora, Iberis violacea R. Br., and Odontarrhena nebrodensis (Tineo) L. Cecchi \& Selvi subsp. nebrodensis from the Madonie Massif (Sicily, Italy).

The study taxa are short-lived perennial herbs, usually woody at the base. The distribution range of $I$. violacea and A. saxatile subsp. saxatile is centered on the Mediterranean basin, extending to continental Europe in the last one, while it is more limited to the western Mediterranean area for A. grandiflora subsp. grandiflora. Finally, O. nebrodensis subsp. nebrodensis is an exclusive endemic taxon to the Madonie Massif. These taxa, which are considered rare in Sicily (Giardina \& al. 2007), occur in rocky habitats on mountain complexes, acting as pioneer plants on screes and debris together with other sparse xerophytes. Their growing sites undergo harsh environmental conditions, with strong winds and intense solar radiation, as well as stress from both seasonal drought and cold. The resulting patchy vegetation type is included within the 8130 Habitat type, i.e., 
'Western Mediterranean and thermophilous screes', listed under the Annex I of the Directive 92/43/EEC. Specifically, A. grandiflora subsp. grandiflora and I. violacea are characteristic taxa of the Arenario-Rumicetum scutati Raimondo 1980 (Linarion purpureae Brullo 1984, Thlaspietea rotundifolii Br.-B1. 1948) (Raimondo \& al. 2004), while O. nebrodensis subsp. nebrodensis is a characteristic taxon of the Cerastio-Astragalion nebrodensis Pignatti \& Nimis ex Brullo 1984 (Brullo 1984).

Seeds were collected in July 2019, between 1300 and $1500 \mathrm{~m}$ a.s.1., in the supraMediterranean belt. Six different constant temperature conditions were tested (from 5 to $\left.30^{\circ} \mathrm{C}\right)$, both in light/dark ( $/ \mathrm{D}, 12 / 12 \mathrm{~h}$ photoperiod) and darkness $(\mathrm{D}, 0 / 24 \mathrm{~h}$ photoperiod), after eight months from seed collection. After such a dry-storage period at room temperature $\left(22 \pm 2{ }^{\circ} \mathrm{C}\right)$, seeds proved to be ready to germinate, without any pre-treatment, over different wide thermal ranges according to different species. Such a feature was highlighted as a frequent trait within the high mountain Mediterranean species (Giménez-Benavides \& al. 2005). This behavior may envisage, if anything, the presence of non-deep physiological dormancy in conditionally dormant fresh seeds, which gradually is released allowing germination from the autumn following seed dispersal to the next spring. Further studies are needed to validate this hypothesis and to better define the timing of germination in the wild.

28. Aethionema saxatile (L.) R.Br. subsp. saxatile (Brassicaceae) (Fig. 1a)

\section{Accession data}

Si: $\quad$ Polizzi Generosa (Palermo), Serre Quacella (WGS84: $37.847111^{\circ} \mathrm{N}, 14.015573^{\circ} \mathrm{E}$ ), screes and debris, $1351 \mathrm{~m}$ a.s.1., $18 \mathrm{Jul}$ 2019, F. Carruggio, M. Castrogiovanni, C. Impelluso, R. Galesi, A. Cristaudo (SiMaSeed/CT/19/135, Catania Germplasm Bank, BGS-CT).

\section{Germination data}

Pre-treatments: no treatment.

Germination medium: 3 sheets of sterilized filter paper (Munktell grade 292, $87 \mathrm{~g} / \mathrm{m}^{2}$ ), imbibed with $6 \mathrm{ml}$ of sterilized distilled water.

Sample size: 100 seeds $(25 \times 4$ replicates $)$.

\begin{tabular}{ccccccc}
\hline Germination & Thermoperiod & $\begin{array}{c}\text { Photoperiod } \\
{[\text { light/dark] }}\end{array}$ & $\mathbf{T}_{\mathbf{1}}[\mathbf{d}]$ & $\mathbf{T}_{50}[\mathbf{d}]$ & $\mathbf{T}_{\max }[\mathbf{d}]$ & MTG [d] \\
\hline $\mathbf{1 0 0 \%}$ & constant $15^{\circ} \mathrm{C}$ & $0 / 24 \mathrm{~h}$ & - & - & - & - \\
\hline $\mathbf{1 0 0} \%$ & constant $20^{\circ} \mathrm{C}$ & $0 / 24 \mathrm{~h}$ & - & - & - & - \\
\hline $\mathbf{1 0 0} \%$ & constant $25^{\circ} \mathrm{C}$ & $0 / 24 \mathrm{~h}$ & - & - & - & - \\
\hline $\mathbf{9 8} \%$ & constant $15^{\circ} \mathrm{C}$ & $12 / 12 \mathrm{~h}$ & 5.0 & 4.5 & 5.0 & 5.0 \\
\hline $\mathbf{9 4 \%}$ & constant $25^{\circ} \mathrm{C}$ & $12 / 12 \mathrm{~h}$ & 3.0 & 3.0 & 4.0 & 3.5 \\
\hline $\mathbf{9 2} \%$ & constant $20^{\circ} \mathrm{C}$ & $12 / 12 \mathrm{~h}$ & 3.0 & 2.5 & 3.0 & 3.0 \\
\hline
\end{tabular}




\section{Observations}

Seeds used in germination tests were taken from dehiscent siliculae. It was possible to verify the seed surface to be covered by specialized cells, producing mucilage and forming a sticky layer (Karaismailoglu 2018), thanks to which seeds can adhere to soil particles.

Seed germination percentage reached high final values, within the thermal range of $15-25^{\circ} \mathrm{C}$, varying from 92 to $100 \%$, regardless of photoperiod conditions. Conversely, outside this thermal range, a negative effect of light on germination was detected at $10^{\circ} \mathrm{C}\left(66 \%\right.$ in L/D vs. $94 \%$ in D), while a positive one at $30^{\circ} \mathrm{C}(92 \%$ in $\mathrm{L} / \mathrm{D}$ vs. $52 \%$ in $\mathrm{D})$. Both temperatures of 20 and $25^{\circ} \mathrm{C}$ provided the best germination performance since these temperature regimes showed the lowest $\mathrm{T}_{50}$ values, namely ca. 2.5-3 days.

The ability to germinate over a wide thermal range, as observed in A. saxatile, is a behavior already detected among high mountain species (Marchand \& Roach 1980). Based on our results, field germination by the autumn following seed dispersal cannot be excluded, even if fresh and short-aged seeds ( $\leq 90$ days) were not tested. Autumn germination would take advantage from the low $\mathrm{T}_{50}$ values that the species showed since young seedlings could have a longer time period for their establishment before snow arrival.

29. Arenaria grandiflora L. subsp. grandiflora (Caryophyllaceae) (Fig. 1b)

\section{Accession data}

Si: Polizzi Generosa (Palermo), Contrada Quacella (WGS84: $37.852792^{\circ} \mathrm{N}$, $14.014092^{\circ} \mathrm{E}$ ), screes and rocky dry grasslands, $1297 \mathrm{~m}$ a.s.1., $17 \mathrm{Jul} 2019, F$. Carruggio, M. Castrogiovanni, C. Impelluso, R. Galesi, A. Cristaudo (SiMaSeed/CT/19/122, Catania Germplasm Bank, BGS-CT).

\section{Germination data}

Pre-treatments: no treatment.

Germination medium: 3 sheets of sterilized filter paper (Munktell grade 292, $87 \mathrm{~g} / \mathrm{m}^{2}$ ), imbibed with $6 \mathrm{ml}$ of sterilized distilled water.

Sample size: 100 seeds $(25 \times 4$ replicates $)$.

\begin{tabular}{ccccccc}
\hline Germination & Thermoperiod & $\begin{array}{c}\text { Photoperiod } \\
{[\text { light/dark] }}\end{array}$ & T $_{1}[\mathbf{d}]$ & T $_{\mathbf{5 0}}[\mathbf{d}]$ & T $_{\max }[\mathbf{d}]$ & MTG [d] \\
\hline $\mathbf{8 8 \%}$ & constant $15^{\circ} \mathrm{C}$ & $12 / 12 \mathrm{~h}$ & 9.0 & 13.0 & 22.0 & 14.1 \\
\hline $\mathbf{8 8 \%}$ & constant $15^{\circ} \mathrm{C}$ & $0 / 24 \mathrm{~h}$ & - & - & - & - \\
\hline $\mathbf{8 4 \%}$ & constant $10^{\circ} \mathrm{C}$ & $0 / 24 \mathrm{~h}$ & - & - & - & - \\
\hline $\mathbf{8 2 \%}$ & constant $20^{\circ} \mathrm{C}$ & $0 / 24 \mathrm{~h}$ & - & - & - & - \\
\hline $\mathbf{7 8 \%}$ & constant $10^{\circ} \mathrm{C}$ & $12 / 12 \mathrm{~h}$ & 8.0 & 9.0 & 16.0 & 9.5 \\
\hline
\end{tabular}




\section{Observations}

Seeds of $A$. grandiflora subsp. grandiflora showed optimal germination values at 10 and $15^{\circ} \mathrm{C}$, both under $\mathrm{L} / \mathrm{D}$ and $\mathrm{D}$ conditions, as well as at $20^{\circ} \mathrm{C}$ under $\mathrm{D}$ regime. Otherwise, a photo-inhibition effect was detected at $20^{\circ} \mathrm{C}(54 \%$ in $\mathrm{L} / \mathrm{D}$ vs. $82 \%$ in D) and germination was poor or very low above this temperature value $(0 \%$ in $\mathrm{L} / \mathrm{D}$ vs. $14 \%$ in $\mathrm{D}$ at $25^{\circ} \mathrm{C} ; 0 \%$ at $30^{\circ} \mathrm{C}$, regardless of photoperiod).

Our data agree with those of Fernàndez-Pascual \& al. (2017) referred to A. grandiflora from Cantabrian Mountains (altitude of the collection site: $1600 \mathrm{~m}$ a.s.1.). The Authors, applying alternating instead of constant temperature conditions on fresh seeds, identified the $14 / 4^{\circ} \mathrm{C}$ regime as the best one compared to $22 / 12$ and $30 / 20^{\circ} \mathrm{C}$. Moreover, obtaining a germination percentage of over $50 \%$ during the 12 -week cold stratification treatment at $3^{\circ} \mathrm{C}$, they suggested the species could have a very low base temperature $\left(\mathrm{T}_{\mathrm{b}}\right)$ rather than a real need for cold stratification. Overall, based on both our data and those of the Iberian researchers, A. grandiflora emerges for its evident 'Mediterranean character' that is planned to protect the species mainly from drought conditions. Further studies are needed to better define the temporal germination window of the species in the wild, assuming that it should be included between autumn and early spring.

30. Iberis violacea $\mathrm{R}$. Br. (Brassicaceae) (Fig. 1c)

\section{Accession data}

Si: Petralia Sottana (Palermo), Contrada Faguara (WGS84: $37.864788^{\circ} \mathrm{N}$, $14.038100^{\circ} \mathrm{E}$ ), screes, $1500 \mathrm{~m}$ a.s.1., $17 \mathrm{Jul}$ 2019, F. Carruggio, M. Castrogiovanni, C. Impelluso, R. Galesi, A. Cristaudo (SiMaSeed/CT/19/130, Catania Germplasm Bank, BGS-CT).

\section{Germination data}

Pre-treatments: no treatment.

Germination medium: 3 sheets of sterilized filter paper (Munktell grade 292, $87 \mathrm{~g} / \mathrm{m}^{2}$ ), imbibed with $6 \mathrm{ml}$ of sterilized distilled water.

Sample size: 100 seeds $(25 \times 4$ replicates $)$.

\begin{tabular}{ccccccc}
\hline Germination & Thermoperiod & $\begin{array}{c}\text { Photoperiod } \\
{[\text { light/dark] }}\end{array}$ & $\mathbf{T}_{\mathbf{1}}[\mathbf{d}]$ & $\mathbf{T}_{\mathbf{5 0}}[\mathbf{d}]$ & $\mathbf{T}_{\mathbf{m a x}}[\mathbf{d}]$ & MTG [d] \\
\hline $\mathbf{9 0 \%}$ & constant $10^{\circ} \mathrm{C}$ & $12 / 12 \mathrm{~h}$ & 5.0 & 15.2 & 14 & 5.8 \\
\hline $\mathbf{8 8 \%}$ & constant $15^{\circ} \mathrm{C}$ & $12 / 12 \mathrm{~h}$ & 3.0 & 4.5 & 16 & 6.0 \\
\hline $\mathbf{8 4 \%}$ & constant $20^{\circ} \mathrm{C}$ & $12 / 12 \mathrm{~h}$ & 3.0 & 3.4 & 10 & 4.0 \\
\hline $\mathbf{8 2 \%}$ & constant $25^{\circ} \mathrm{C}$ & $12 / 12 \mathrm{~h}$ & 3.0 & 4.6 & 16 & 6.2 \\
\hline
\end{tabular}




\section{Observations}

Seeds of I. violacea showed high germination values $(>80 \%)$ throughout the range 10$20^{\circ} \mathrm{C}$, both under L/D and D conditions. Germination performance slightly decreased under $\mathrm{D}$ condition at $25^{\circ} \mathrm{C}(68 \%)$ and markedly fell at $30^{\circ} \mathrm{C}(30$ and $8 \%$, in L/D and D conditions, respectively). No significant temperature effect on germination speed was detected in the range $15-25^{\circ} \mathrm{C}$, within which the lowest $\mathrm{T}_{50}$ values were registered (ca. 4 days). Overall, I. violacea showed a comparable germination behavior to that of $A$. saxatile, although the species has a lower ceiling temperature.

31. Odontarrhena nebrodensis (Tineo) L.Cecchi \& Selvi subsp. nebrodensis (Brassicaceae) (Fig. 1d)

\section{Accession data}

Si: Polizzi Generosa (Palermo), Contrada Quacella (WGS84: $37.852792^{\circ} \mathrm{N}$, $\left.14.014092^{\circ} \mathrm{E}\right)$, screes and rocky dry grasslands, $1297 \mathrm{~m}$ a.s.l., $17 \mathrm{Jul} 2019, F$. Carruggio, M. Castrogiovanni, C. Impelluso, R. Galesi, A. Cristaudo (SiMaSeed/CT/19/124, Catania Germplasm Bank, BGS-CT).

\section{Germination data}

Pre-treatments: no treatment.

Germination medium: 3 sheets of sterilized filter paper (Munktell grade 292, $87 \mathrm{~g} / \mathrm{m}^{2}$ ), imbibed with $6 \mathrm{ml}$ of sterilized distilled water.

Sample size: 100 seeds $(25 \times 4$ replicates $)$.

\begin{tabular}{ccccccc}
\hline Germination & Thermoperiod & $\begin{array}{c}\text { Photoperiod } \\
\text { [light/dark] }\end{array}$ & $\mathbf{T}_{\mathbf{1}}[\mathbf{d}]$ & $\mathbf{T}_{\mathbf{5 0}}[\mathbf{d}]$ & $\mathbf{T}_{\mathbf{m a x}}[\mathbf{d}]$ & MTG [d] \\
\hline $\mathbf{9 8 \%}$ & constant $15^{\circ} \mathrm{C}$ & $12 / 12 \mathrm{~h}$ & 1.0 & 1.3 & 5 & 2.0 \\
\hline $\mathbf{9 8 \%}$ & constant $10^{\circ} \mathrm{C}$ & $12 / 12 \mathrm{~h}$ & 3.0 & 3.4 & 6 & 4.3 \\
\hline $\mathbf{9 6 \%}$ & constant $20^{\circ} \mathrm{C}$ & $12 / 12 \mathrm{~h}$ & 1.0 & 0.5 & 6 & 1.3 \\
\hline $\mathbf{9 4 \%}$ & constant $25^{\circ} \mathrm{C}$ & $12 / 12 \mathrm{~h}$ & 1.0 & 0.5 & 6 & 1.1 \\
\hline $\mathbf{9 2 \%}$ & constant $30^{\circ} \mathrm{C}$ & $12 / 12 \mathrm{~h}$ & 1.0 & 0.6 & 15 & 2.2 \\
\hline $\mathbf{9 2 \%}$ & constant $5^{\circ} \mathrm{C}$ & $12 / 12 \mathrm{~h}$ & 6.0 & 7.6 & 16 & 9.0 \\
\hline
\end{tabular}

\section{Observations}

High germination percentage $(>90 \%)$ was registered throughout the entire temperature range tested, regardless of photoperiod. The germination process was notably fast within the range $20-30^{\circ} \mathrm{C}\left(\mathrm{T}_{50}<1\right.$ day) but quite fast also at $10-15^{\circ} \mathrm{C}$. Such behavior may allow germination to occur from the autumn following seed dispersal to the next spring. 


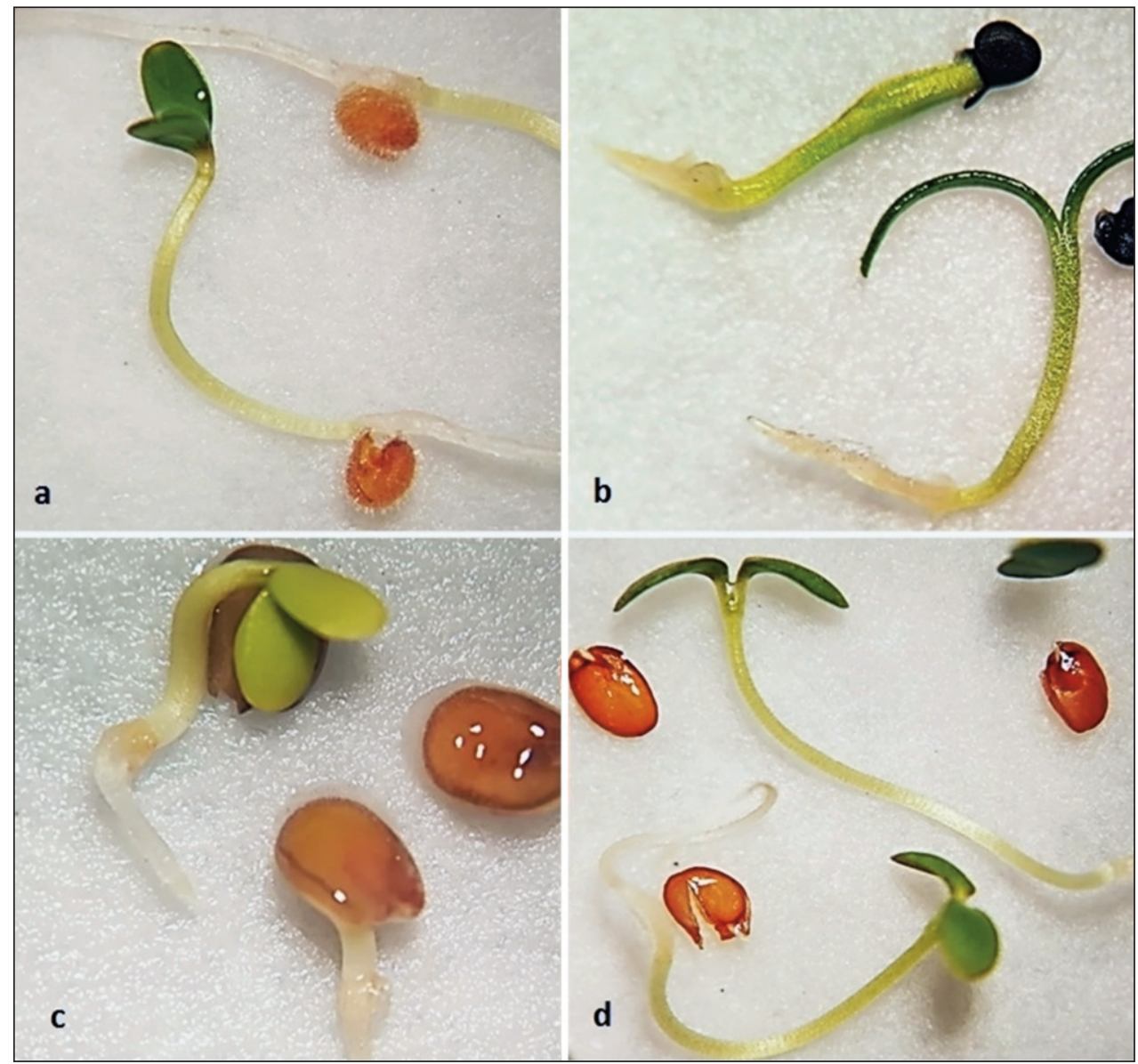

Fig. 1. Germinated seeds and seedlings: a, Aethionema saxatile subsp. saxatile; b, Arenaria grandiflora subsp. grandiflora; $\mathbf{c}$, Iberis violacea; $\mathbf{d}$, Odontarrhena nebrodensis subsp. nebrodensis.

\section{References}

Brullo, S. 1984: Contributo alla conoscenza della vegetazione delle Madonie (Sicilia settentrionale). - Boll. Acc. Gioenia Sci. Nat. Catania 16: 351-420.

Fernández-Pascual, E., Jiménez-Alfaro, B. \& Bueno, Á. 2017: Comparative seed germination traits in alpine and subalpine grasslands: higher elevations are associated with warmer germination temperatures. - Pl. Biol. 19: 32-40. http://dx.doi.org/10.1111/plb.12472

Giardina, G., Raimondo, F. M. \& Spadaro, V. 2007: A catalogue of plants growing in Sicily. Bocconea 20: 5-582.

Giménez-Benavides, L., Escudero, A., Péres-Garcìa, F. 2005: Seed germination of high mountain Mediterranean species: altitudinal, interpopulation and interannual variability. - Ecol. Res. 20: 433-444. http://dx.doi.org/10.1007/s11284-005-0059-4 
Karaismailoglu, M. 2018: Seed mucilage content in Aethionema W.T. Aiton species and their significance in systematic and ecological aspects. - Bangladesh J. Bot. 47: 445-449. http://dx.doi.org/10.3329/bjb.v47i3.38682

Marchand, P. \& Roach, D. 1980: Reproductive strategies of pioneering alpine species: seed production, dispersal, and germination. - Arct. Antarct. Alp. Res. 12: 137-146. http://dx.doi.org/10.1080/00040851.1980.12004173

Raimondo, F., Schicchi, R. \& Surano, N. 2004: Carta del paesaggio e della biodiversità vegetale del parco delle Madonie (Sicilia). - Naturalista Sicil., s.4, 28: 71-137.

Address of the authors:

Francesca Carruggio, Maria Castrogiovanni, Carmen Impelluso, Antonia Cristaudo*,

Catania Germplasm Bank, Department of Biological, Geological and Environmental Sciences, Catania University, Catania, Italy. E-mail: acristau@unict.it

*RIBES, Rete Italiana Banche del germoplasma per la conservazione Ex Situ della flora italiana, www.reteribes.it 